\title{
The inner workings of early-type galaxies: cores, nuclei and supermassive black holes
}

\author{
Laura Ferrarese and Patrick Côté \\ National Research Council of Canada, Herzberg Institute of Astrophysics, 5071 West Saanich \\ Road, Victoria, BC, V9E 2E7, Canada \\ email: laura.ferrarese@nrc-cnrc.gc.ca, patrick.cote@nrc-cnrc.gc.ca
}

\begin{abstract}
Recent years have seen dramatic progress in the study of the core and nuclear properties of galaxies. The structure of the cores has been shown to vary methodically with global and nuclear properties, as cores respond to the mechanisms by which galaxies form/evolve. The dynamical centers of galaxies have been found capable of hosting two seemingly disparate objects: supermassive black holes (SBHs) and compact stellar nuclei. In a drastic departure from previous beliefs, it has been discovered that both structures are common: galaxies lacking SBHs and/or stellar nuclei are the exception, rather than the norm. This review explores the connection between cores, SBHs and stellar nuclei in early-type galaxies, as revealed by the ACS Virgo Cluster Survey.
\end{abstract}

Keywords. Galaxies: elliptical and lenticular, cD - galaxies: dwarf - galaxies: fundamental parameters - galaxies: photometry - galaxies: structure - galaxies: nuclei - galaxies: bulges

\section{Introduction}

Cores - a term which we will use loosely to describe the central few hundred parsecs of galaxies - act as recording devices of the process of galaxy evolution. Because dynamical timescales are shorter than elsewhere in the galaxy, the morphology, dynamics, and stellar populations of the core regions contain fossil records of the gas, dust and stellar systems (satellite galaxies, star clusters, etc) that were drawn to the bottom of the gravitational potential well over cosmic timescales. Moreover, it has now become clear that the core and global properties of galaxies are linked through a number of intriguing scaling relations. In particular, those involving supermassive black holes (SBHs) - which, in high-luminosity galaxies at least, appear to be a generic subcomponent of the cores - underscore the importance of nuclear feedback in galaxy evolution (Ferrarese \& Merritt 2000; Gebhardt et al. 2000; Graham et al. 2001; Ferrarese 2002; Haring \& Rix 2004).

The study of galactic cores received a tremendous push forward with the deployment of the Hubble Space Telescope. HST images brought into focus a plethora of structural features, including nuclear stellar disks, bars, "evacuated" regions (possibly scoured out by the evolution of SBH binaries), and an entire spectrum of dust features, from small irregular patches to large, highly organized disks. In early-type galaxies, cores were found to fall into two distinct classes: those with a shallow surface brightness profile, and those whose surface brightness kept increasing, in roughly a power-law fashion, to the innermost radius accessible given the resolution limit of the instrument (Ferrarese et al. 1994; Lauer et al. 1995,2005; Ravindranath et al. 1996; Rest et al. 2001). Galaxies falling into the first class have come to be known (somewhat unfortunately) as "core" galaxies, and those falling into the second class as "power-law". The division between the two classes was found to correlate neatly with galaxy luminosity, with core galaxies being exclusively bright giant ellipticals, while fainter galaxies are (with few exceptions) classified 
as power-laws. The stark separation between the two classes has been attributed to differing formation/evolutionary histories. Power-law galaxies have been claimed to be the result of dissipation during galaxy formation, with some authors further claiming that all power-law galaxies host stellar disks; while core-galaxies are believed to be the result of the merging of fainter (power-law) galaxies, and of their central SBHs.

SBHs have now been detected dynamically in roughly three dozen galaxies (see Ferrarese \& Ford 2005 for a review). Indeed, balancing the SBH mass function from the QSO epoch to the present day requires virtually all local galaxies brighter than a few $0.1 L^{*}$ to host a SBH (e.g. Shankar et al. 2004; Marconi et al. 2004). Recent observations, however, have made it clear that SBHs are not the only objects to enjoy a privileged position at a galaxy's dynamical center. Stellar nuclei, or nuclear star clusters, have recently been detected in a large fraction ( $70 \%$ to $90 \%$ ) of galaxies of all Hubble types and luminosities (Böker et al. 2002; Lotz et al. 2004; Grant et al. 2005). Follow-up spectroscopy of stellar nuclei in spiral galaxies (Walcher et al. 2005, 2006; Rossa et al. 2006) has shown them to be massive, dense objects akin to compact star clusters. Luminosity-weighted ages range from 10 Myr to $10 \mathrm{Gyr}$, younger than the age of the galactic disk, and with the younger clusters found preferentially in the later type spirals.

This review explores the connection between cores, nuclei and supermassive black holes in light of recent results from the ACS Virgo Cluster Survey (ACSVCS), the largest HST imaging survey to date of early-type galaxies in the local universe. This survey was designed specifically to provide an unbiased characterization of the core structure of early-type galaxies using well understood selection criteria.

\section{The ACS Virgo Cluster Survey}

The ACSVCS (Côté et al. 2004) consists of HST imaging for 100 members of the Virgo Cluster, supplemented by imaging and spectroscopy from WFPC2, Chandra, Spitzer, Keck and KPNO. The program galaxies span a range of $\approx 460$ in $B$-band luminosity and have early-type morphologies: $\mathrm{E}, \mathrm{S} 0, \mathrm{dE}, \mathrm{dE}, \mathrm{N}$ or dS0. All images were taken with the Advanced Camera for Surveys (ACS; Ford et al. 1998) using a filter combination roughly equivalent to the $g$ and $z$ bands in the SDSS photometric system. The images cover a $\approx$ $200^{\prime \prime} \times 200^{\prime \prime}$ field with $\approx 0^{\prime \prime} .1$ resolution ( $\approx 8 \mathrm{pc}$ at the distance of Virgo, $\left.D=16.5 \mathrm{Mpc}\right)$.

This review summarizes results from the subset of ACSVCS papers which deal with the morphology, isophotal parameters and surface brightness profiles for early-type galaxies (Ferrarese et al. 2006a), their central nuclei (Côté et al. 2006) and scaling relations for nuclei and SBHs (Ferrarese et al. 2006b). Other ACSVCS papers have discussed the data reduction pipeline (Jordán et al. 2004a), the connection between low-mass X-ray binaries and globular clusters (Jordán et al. 2004b), the measurement and calibration of surface brightness fluctuation distances (Mei et al. 2005ab), the connection between globular clusters and ultra-compact dwarf galaxies (Haşegan et al. 2005), the luminosity function, color distributions and half-light radii of globular clusters (Jordán et al. 2005, 2006; Peng et al. 2006a), and diffuse star clusters (Peng et al. 2006b).

\section{The core structure of early-type galaxies}

Over the three-decade radial range between a few tens of parsecs and several kiloparsecs (i.e. to the largest radii covered by the ACSVCS images), the surface brightness profiles of the ACSVCS early-type galaxies are well described by a simple Sérsic model (Sérsic 1968) with index $n$ increasing steadily with galaxy luminosity. By contrast, a "Nuker" model (Lauer et al. 1995; 2005) fails to reproduce the curved surface brightness profiles that real 
galaxies exhibit and often grossly overestimates the luminosity at large and intermediate radii. Notable, and systematic, deviations from a Sérsic model are however registered in the innermost regions. For eight of the 10 brightest galaxies $\left(M_{B} \lesssim-20.3\right)$ the measured inner profiles (typically within $0^{\prime \prime} .5$ to $2^{\prime \prime} .5$, corresponding to 40 to $200 \mathrm{pc}$ ) are shallower than expected based on an inward extrapolation of the Sérsic model constrained by the region beyond. For these galaxies, the surface brightness profile is best fitted by joining the outer Sérsic profile to an inner, shallower, power-law component (such composite models are referred to as "core-Sérsic", Graham et al. 2003; Trujillo et al. 2004),

The opposite is seen in fainter galaxies, where $\approx 80 \%$ of the sample galaxies show an upturn, or inflection, in the surface brightness profile within (typically) the innermost few tens of parsec region (see Figure 1 of P. Côté, these proceedings). The upturn signals the presence of a stellar nucleus that is most likely structurally distinct from the main body of the underlying galaxy. When a nucleus is present, the inner surface brightness is, by definition, larger than the inward extrapolation of the outer Sérsic model.

The picture that has emerged from the ACSVCS is therefore one in which, in moving down the luminosity function from giant to dwarf early-type galaxies, the innermost 100parsec region undergoes a systematic and smooth transition from light (mass) "deficit" (relative to the overall best fitting Sérsic model) to light "excess". Although the subset of ACSVCS "core-Sérsic" galaxies coincides with the galaxies that were classified as "cores" in previous investigations, there are critical differences between our study and the ones that preceded it. Compared to previous work, the ACSVCS has emphasized the role of stellar nuclei; the fact that the frequency, luminosities and sizes of the ACSVCS nuclei are in remarkable agreement with those measured (using different techniques and assumptions) by recent independent surveys in both early and late type galaxies, supports the robustness of the ACSVCS analysis. Recognizing the nuclei as separate components has allowed us to revisit the issue of the division of early-type galaxies into "core" and "power-law" types. Such division was based on the fact that the distribution of the logarithmic slopes, $\gamma=-d \log I / d \log r$, of the inner surface brightness profile had been found to show various degrees of bimodality. Such bimodality is absent in the $\gamma$ distribution of the ACSVCS galaxies.

In agreement with previous studies, in galaxies brighter than $M_{B} \approx-20.3, \gamma$ is indeed found to decrease with galaxy luminosity, while the opposite trend is seen for fainter galaxies, however, the transition is smooth, rather than abrupt. In a further departure from previous studies, we find that the low- $\gamma$ end of the distribution (corresponding to the galaxies with the shallowest surface brightness profiles) is occupied mostly by the faintest dwarf systems, rather than by the brightest giant ellipticals. We note here that the absence of a bimodal behaviour in $\gamma$ does not automatically invalidate a picture in which brighter galaxies evolve mainly through merging while fainter systems are largely left untouched. Indeed, such picture does not necessarily explain the perceived stark separation of galaxies in "core" and "power-law" types for which it was formulated. The extent to which structural parameters are compromised by merging of galaxies (and their supermassive black holes) depends on the the masses of the progenitors (e.g., Bournaud et al. 2005; Milosavljevic \& Merritt 2001); given a continuous distribution for the latter, combined with a galaxy luminosity function heavily biased towards low-mass systems, allows for the possibility of a smooth transition between progenitors and merger products.

\section{Compact stellar nuclei in the ACSVCS}

At the outset of the ACSVCS, it was known that at least $\approx 25 \%$ of the program galaxies contained nuclei, based on ground-based, photographic classifications from the 
VCC (Binggeli et al. 1985). Stellar nuclei in the ACSVCS images were identified using a variety of indicators, including direct inspection of the ACS frames, color changes in the $g-z$ color images, and sudden upturns in the surface brightness profiles. Based on these criteria, 60 to $80 \%$ of ACSVCS galaxies host stellar nuclei (with the precise fraction depending on galaxy magnitude), in line with the fraction reported in both spiral and elliptical galaxies based on recent high-resolution surveys (Carollo, Stiavelli \& Mack 1998; Matthews et al. 1999; Böker et al. 2002, 2004; Balcells et al. 2003; Lotz et al. 2004; Graham \& Guzman 2003; Grant et al. 2005), but a factor $~ 3$ higher than expected based on the VCC.

Our analysis shows that surface brightness selection biases in the VCC data are largely responsible for the difference. In galaxies with central $g$-band surface brightnesses lower than $\approx 20.5 \mathrm{mag} \operatorname{arcsec}^{-2}$, the agreement between the ACSVCS and VCC is nearly perfect, while above $19.5 \mathrm{mag} \operatorname{arcsec}^{-2}$, virtually all nuclei were missed by the groundbased survey. Selection effects might, of course, still be at work in the ACSVCS sample, implying that our estimate for the frequency of nucleation, $f_{n} \approx 60-80 \%$, is almost certainly a lower limit on the true frequency. As will be discussed shortly, the luminosity and half-light radii of stellar nuclei correlate strongly with the magnitude of the host galaxy; it is therefore possible, for each galaxy classified as non-nucleated, to determine whether a nucleus, if present, could have gone undetected.

Based on these tests, with very few exceptions, the only galaxies for which the existence of a nucleus can be confidently excluded are those brighter than $M_{B} \approx-20.3$ mag. These are the same galaxies with central light "deficits" for which the surface brightness profiles are well represented by "core-Sérsic" rather than Sérsic models (§3).

Scaling relations for stellar nuclei. For 51 galaxies in the ACSVCS the sharp upturn in the surface brightness within $\approx 1^{\prime \prime}$ is conspicuous enough that a measurement of the nucleus' photometric and structural parameters is possible. These parameters are recovered by adding a central King model (King 1966) to the underlying Sérsic component when fitting the brightness profile.

The luminosity function of nuclei follows a Gaussian distribution with dispersion in the range $1.5-1.8 \mathrm{mag}$ and peak absolute $g$-band magnitude $\approx-10.7$ mag, a factor $\approx 25 \times$ brighter than the peak of the globular cluster luminosity function. With a halfdozen exceptions, nuclei in the ACSVCS galaxies are spatially resolved (thereby ruling out a non-thermal AGN origin), with individual sizes ranging from $62 \mathrm{pc}$ down to the resolution limit of $2 \mathrm{pc}$, and a median half-light radius of $\left\langle r_{h}\right\rangle=4.2 \mathrm{pc}$. Unlike globular clusters, for which size is independent of magnitude, nuclear sizes are found to scale with luminosity according to the relation $r_{h} \propto \mathcal{L}^{0.50 \pm 0.03}$ (Figure 1, left panel).

One of the most popular models for the formation of nuclei posits that globular clusters are drawn to the bottom of the potential well by dynamical friction, where they then coalesce (e.g. Tremaine et al. 1975). While the size-magnitude relation observed for the ACSVCS nuclei is consistent with the prediction of such model (Bekki et al. 2004), a more complex picture is suggested by the observations that nuclei, again unlike globular clusters, display a color-magnitude relation (Figure 1, right panel). Monte Carlo simulations show that mergers of globular clusters through dynamical friction are unable to explain the observed color-magnitude relation; indeed the existence of this relation suggests that the chemical enrichment of nuclei is governed by local or internal factors, along the lines of the various "gas accretion" models (e.g. Mihos \& Hernquist 1996). Note that the nuclei's color-magnitude relation is better defined for galaxies fainter than $M_{B} \approx-17.6 \mathrm{mag}$, while the nuclei belonging to brighter galaxies frequently show very red colors, $(g-z) \sim 1.5$. If confirmed (measurements are more uncertain for these nuclei, 

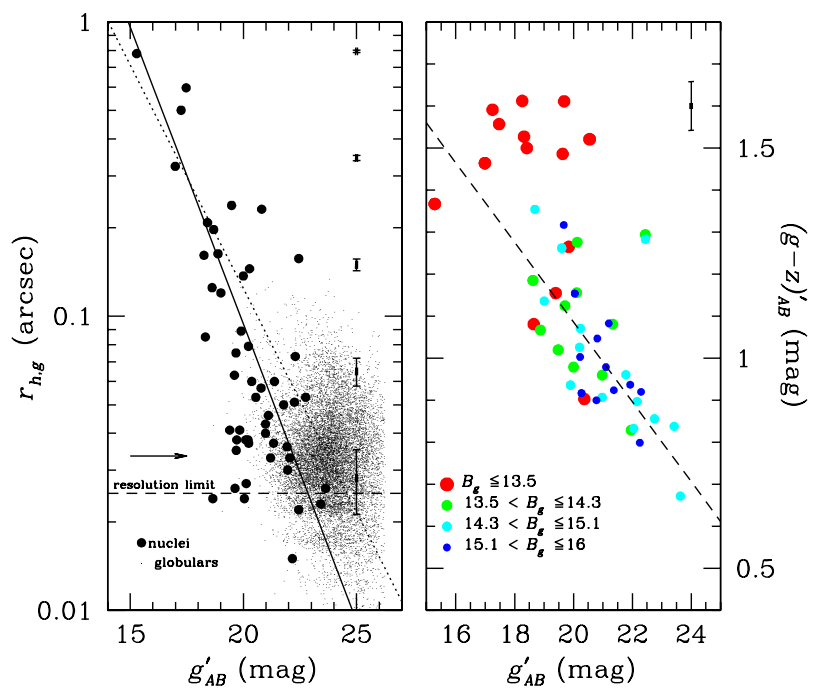

Figure 1. Left panel: the size-magnitude relation, in the $g$-band, for the 51 ACSVCS nuclei for which structural parameters could be measured (solid circles) and the sample of globular clusters from Jordán et al. (2005) (points). Typical error-bars for the nuclear sizes are shown in the right hand side of the panel. The arrow shows the "universal" half-light radius of 0 ".033 $(\approx 2.7 \mathrm{pc}$ ) for globular clusters in Virgo (Jordán et al. 2005), while the dashed line shows a conservative estimate for the resolution limit of the ACS images. The solid diagonal line shows the best fitting relation for the nuclei $\left(r_{h} \propto L^{0.5}\right)$, while the dotted line shows the prediction of the globular cluster merging model of Bekki et al. (2004). Right panel: color-magnitude diagram for the ACSVCS nuclei. The size of the symbol for the nuclei is proportional to the magnitude of the host galaxy, as shown in the legend. The dashed line shows the best fit relation for the nuclei of galaxies fainter than $B_{T}=13.5 \mathrm{mag}$.

due to the high underlying galaxy surface brightness), this observation might suggest that these nuclei may constitute a separate class following a different formation route.

A third model, namely nuclear formation through two-body relaxation around a black hole, is inconsistent with the observation that nuclei are spatially resolved in most of the ACSVCS galaxies. Nuclei formed through this mechanism are predicted to extend to $\approx$ 1/5th of the SBH sphere of influence (e.g. Merritt \& Szell 2005), and would therefore be spatially unresolved by the ACS in all of the ACSVCS galaxies, in clear contradiction with our observations. Finally, we note that the luminosity function and size distribution of the ACSVCS nuclei shows remarkable agreement with those of the "nuclear star clusters" detected in spiral galaxies (Böker et al. 2002, 2004). This points to a formation mechanism for the nuclei that is largely independent on both intrinsic and extrinsic factors, such as host magnitude and Hubble type, and immediate environment.

\section{Stellar Nuclei and Supermassive Black Holes}

The ubiquity of SBHs and stellar nuclei, and their unique location at the dynamical centers of galaxies, are reasons to suspect that a connection between the two might exist. The ACSVCS data strongly support this view (Ferrarese et al. 2006b). The left panel of Figure 2 shows a recent characterization of the relation between the masses of SBHs (black circles) and the stellar velocity dispersion of the host bulge, originally discovered by Ferrarese \& Merritt (2000) and Gebhardt et al. (2000).

The $\mathcal{M}-\sigma$ relation is one of the tightest, and therefore most fundamental, of the scaling relations for SBHs, and has been used extensively to constrain the joint evolution of SBHs and galaxies (e.g., Haehnelt 2004 and references therein). The ACSVCS stellar 


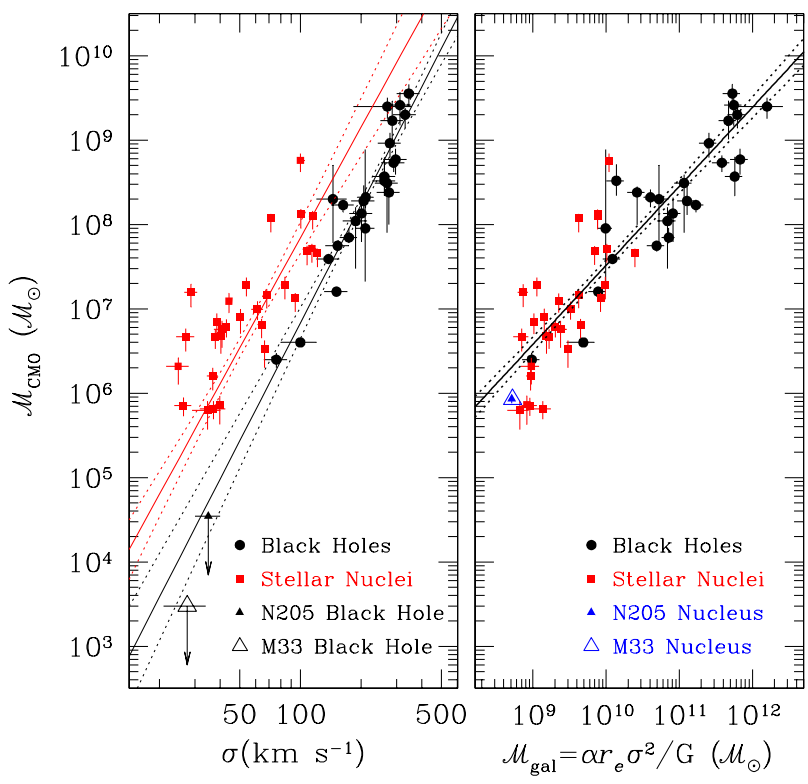

Figure 2. Left panel: mass of the CMO (stellar nuclei as red squares and SBHs as black circles) plotted against the velocity dispersion of the host galaxy. The solid red and black lines show the best fits to the nuclei and SBH samples, respectively, with $1 \sigma$ confidence levels shown by the dotted lines. Right panel: CMO mass plotted against galaxy virial mass. The solid line is the fit obtained for the combined nuclei and SBH sample.

nuclei (shown as red squares) obey an $\mathcal{M}-\sigma$ relation with the same slope, although different normalization, as the one defined by SBHs. This is a notable and unexpected finding, suggesting close similarities in the formation and evolutionary history of these two radically different structures (McLaughlin et al. 2006). Furthermore, when $\sigma$ is combined with the effective radius $r_{e}$ to produce a measure of the galaxy's virial mass, $\mathcal{M}_{\text {gal }} \propto$ $\sigma^{2} r_{e} / G$, SBHs and stellar nuclei are found to obey an identical $\mathcal{M}-\mathcal{M}_{\text {gal }}$ relation (right panel of Figure 2; see also Côté et al. 2006). Remarkably, the same relation is also found to hold in spiral galaxies (Rossa et al. 2006) and to extend to dEs as faint as $M_{B} \approx-11.7$ mag (Wehner \& Harris 2006).

These findings can be summarized as follows: a constant fraction, $\mathcal{M}_{\mathrm{CMO}} / \mathcal{M}_{\text {gal }} \approx$ $0.3 \%$, of a galaxy total mass is used in the formation of a nuclear structure, or "central massive object" (CMO). This holds true for galaxies spanning a factor $10^{4} \mathrm{M}_{\odot}$ in mass, all Hubble types, luminosities and environments. In spite of their obviously different nature, SBHs and stellar nuclei may be nothing but complementary incarnations of CMOs they likely share a common formation mechanism and follow a similar evolutionary path throughout their host galaxy's history. From the perspective of a theoretical framework of galaxy evolution, the commonalities between SBHs and stellar nuclei imply that both are equally important constraints on galaxy formation models: as the characterization of SBHs has been instrumental in furthering our understanding of galaxy evolution (via AGN feedback), so too promises to be the characterization of stellar nuclei (via superwinds and stellar feedback).

Several questions remain unanswered at this stage. Perhaps the most intriguing is whether the formation of SBHs and stellar nuclei are mutually exclusive. Nuclei are not present in the brightest ACSVCS galaxies, the prototypical objects in which SBHs are expected to reside, and for which a "mass deficit" has been attributed to the evolution 
of supermassive black hole binaries (Milosavljevic \& Merritt 2001). At the other extreme of the luminosity range spanned by the ACSVCS galaxies, NGC205 and M33, for which there is no evidence of a SBH (Merritt et al. 2001; Gebhardt et al. 2001; Valluri et al. 2003), host stellar nuclei that follow the same scaling relations as the nuclei detected in the ACSVCS galaxies (Figure 2, right panel).

It is possible that nuclei form in every galaxy, but are subsequently destroyed in the brightest system as a consequence of the evolution of SBH binaries. Alternatively, it is possible that collapse to a $\mathrm{SBH}$ takes place preferentially in the brightest galaxies, while in fainter systems, the formation of a stellar nucleus is the most common outcome. In the latter case, nuclei could represent "failed black holes" - low-mass counterparts of the SBHs detected in the brightest galaxies.

The ACSVCS collaboration is currently pursuing several programs aimed at studying the dynamics and stellar population of the ACSVCS galaxies and nuclei; a similar investigation is underway for a sample of 43 early-type galaxies in the Fornax Cluster (Jordán et al. $2006 \mathrm{~b}$, in preparation). These projects promise to shed further light on the core structure of early-type galaxies, their nuclei and their relationships to SBHs.

\section{Appendix}

In a recent submission to astro-ph (astro-ph/0609762), Lauer et al. have reiterated their claim for a bimodal distribution of the inner profile slopes $\gamma$, and have questioned the reliability of the ACSVCS results. Their assertions are based on a heterogeneous sample of early-type galaxies - at widely differing distances and observed with a variety of instruments/filters/spatial resolutions — whose surface brightness profiles have been parametrized as "Nuker" laws (which has been shown to produce biased results and to be inadequate in describing the full extent of the surface brightness profiles of early-type galaxies; e.g., Graham et al. 2003).

In their posting, Lauer et al. further claim that the parametric form used by Ferrarese et al. (2006a) to fit the profiles of the ACSVCS galaxies forces the introduction of ad hoc stellar nuclei. These claims are difficult to understand in light of the fact that Lauer et al. themselves define nuclei as an inner excess against an adopted (Nuker) model, despite the fact that such models fail to match the data at small and large scales. More details on the gross misrepresentation of the ACSVCS data and analysis by Lauer et al. can be found on the ACSVCS website: http://www.cadc.hia.nrc.gc.ca/community/ACSVCS/

\section{Acknowledgements}

More rewarding than the results themselves has been to work with a great team. The friendship, hard work and dedication of each member of the ACSVCS team is very gratefully acknowledged.

\section{References}

Bekki, K., et al. 2004, ApJ, 610, L13

Best, P.N. et al. 2006, MNRAS, 368, 67

Binggeli, B., Sandage A. \& Tammann G. A. 1985, AJ, 90, 1681

Böker, T. et al. 2002, AJ, 123, 1389

Böker, T. et al. 2004, AJ, 127, 105

Bournaud, F., Jog, C. J. \& Combes, F. 2005, A\&A, 437, 69

Côté, P. et al. 2004, ApJS, 153, 223

Côté, P. et al. 2006, ApJS, 165, 57

Ferrarese, L. \& Merritt, D. 2000, ApJL, 539, 9

Ferrarese, L. 2002, ApJ, 578, 90

Ferrarese, L., Ford, H. C. 2005, Sp.Sc.Rev.,116, 523 
Ferrarese, L. et al. 1994, AJ, 108, 1598

Ferrarese, L. et al. 2006a, ApJS, 164, 334

Ferrarese, L. et al. 2006b, ApJL, 644, L21

Gebhardt, K. et al. 2000, ApJ, 539, L13

Graham, A. et al. 2001, ApJ, 563, L11

Graham, A. W., Erwin, P., Trujillo, I. \& Asensio, R. A. 2003, AJ, 125, 2951

Grant, N. I. et al. 2005, MNRAS, 363, 1019

Haehnelt, M., 2004, in oevolution of Black Holes and Galaxies 405

Haring, N., Rix, H.-W. 2004, ApJ, 604, 89

Hasegan, M. et al. 2005, ApJ, 627, 203

Jordán, A. et al. 2004a, ApJS, 154, 509

Jordán, A. et al. 2004b, ApJ, 613, 279

Jordán, A. et al. 2005, ApJ, 634,1002

Jordán, A. et al. 2006, ApJL, in press

Lauer, T. et al. 1995, AJ, 110, 2622

Lauer, T. et al. 2005, AJ, 129, 2138

Lotz, J., Miller, B. W \& Ferguson, H. C. 2004, ApJ, 613, 262

Marconi, A. et al. 2004, MNRAS, 351, 169

McLaughlin, D. E., King, A. R. \& Nayakshin, S. 2006, ApJL, in press (astro-ph/0608521)

Mei, S. et al. 2005a, ApJS, 156, 113

Mei, S. et al. 2005b, ApJ, 625, 121

Merritt, D., Ferrarese, L. \& Joseph, C. 2001, Science, 293, 1116

Merritt, D. \& Szell, A. 2005, ApJ, 648, 890

Milosavljevic, M. \& Merritt, D. 2003, ApJ, 596, 860

Mihos, C. \& Hernquist, L. 1996, ApJ, 464, 461

Peng, E. W. et al. 2006a, ApJ, 639, 95.

Peng, E.W. et al. 2006b, ApJ, 639, 838.

Ravindranath, S. et al. 2001, AJ, 122, 653

Rest, A. et al. 2001, AJ, 121, 2431

Rossa, J. et al. 2006, AJ, 132, 1074

Shankar, F. et al. 2004, MNRAS, 354, 1020

Tremaine, S. D., Ostriker, J. P. \& Spitzer, L., Jr. 1975, ApJ, 196, 407

Valluri, M., Ferrarese, L., Merritt, D. \& Joseph, C. 2005, ApJ, 628, 137

Walcher, C. J. et al. 2005, ApJ, 618, 327

Walcher, C. J. et al. 2006, ApJ, in press (astro-ph/0604138)

Wehner, E. \& Harris, W. E. 2006, ApJL, 644, L17

Deborah Dultzin-Hacyan: Could you explain what kind of (long-slit) spectrum one has in the nucleus?

LAURA Ferrarese: Indeed we use long-slit spectra. Even with HST these nuclei are barely resolved. Therefore, separating nuclear spectra from the galaxy is rather demanding. For the time being the nucleus definition is based on morphology.

Mitchell Begelman: The SLOAN survey of type 2 AGNs seems to find a transitions galaxy mass below which there is no activity. How does your transition mass between nuclei and black holes compare to that mass?

LAURA FERRARESE: It is of about the same value, a few times $10^{10} M_{\odot}$. 